\title{
HIPERCOLESTEROLEMIA FAMILIAR Y DIAGNÓSTICO. REVISIÓN DE LA PRODUCCIÓN CIENTÍFICA CON APOYO DE INDICADORES BIBLIOMÉTRICOS.
}

\author{
FAMILIAL HYPERCHOLESTEROLEMIA AND DIAGNOSIS. REVIEW OF \\ SCIENTIFIC PRODUCTION SUPPORTED BY BIBLIOMETRIC INDICATORS.
}

Autor 1 - Luz Mery Bernal

Biol. MSc. PhD. Escuela de Ciencias de la Salud. Universidad Nacional Abierta y a Distancia.

ORCID https://orcid.org/0000-0003-1271-5903

email. luz.bernal@unad.edu.co

Autor 2 - Claudia Marcela Sabogal

Odont. MSc. Escuela de Ciencias de la Salud. Universidad Nacional Abierta y a Distancia.

ORCID https://orcid.org/0000-0003-0928-4153

email. claudia.sabogal@unad.edu.co

Autor 3 - Greizy López Leal.

Bact. PhD. Instituto de Genética Humana, Pontificia Universidad Javeriana, Bogotá.

ORCID https://orcid.org/0000-0001-9380-0792

email.greizy.lopez@javeriana.edu.co

Autor 4 - Nancy Yaneth Gelvez.

Bact. MSc. Instituto de Genética Humana, Pontificia Universidad Javeriana, Bogotá.

ORCID https://orcid.org/0000-0002-3925-1451

email. nancy.gelvez@javeriana.edu.co

Autor 5 - Gustavo Jaimes

Odont. MSc. Escuela de Ciencias de la Salud. Universidad Nacional Abierta y a Distancia.

ORCID https://orcid.org/0000-0003-3918-3679

Autor 6 - Marta Lucía Tamayo F.

MD. MSc. Instituto de Genética Humana, Pontificia Universidad Javeriana, Bogotá.

ORCID https://orcid.org/0000-0001-8297-3970

email.mtamayo@javeriana.edu.co 


\section{RESUMEN.}

La Hipercolesterolemia Familiar (HF) es un trastorno genético que provoca el aumento del colesterol en la sangre y un incremento importante de desarrollo de ateroesclerosis. Objetivo: establecer el estado de la investigación en el tema de hipercolesterolemia familiar, revisar los principales documentos desarrollados en relación con la temática y explorar los posibles vacíos de conocimiento que surjan de este ejercicio académico con respecto al diagnóstico. Métodos: Se realizó una revisión con indicadores bibliométricos desde artículos de la base de datos de Web of Science, para el análisis se utilizaron los programas libres VOSviewer y Bibliometrix aplicación de R. Adicionalmente se revisaron los textos más citados. Resultados: Un total de 1102 artículos fueron hallados mediante los criterios de inclusión (HF y diagnóstico). Los autores más citados fueron Watts, GF. de Australia con $n=32$ publicaciones y 1279 citas, seguido por Kastelein, JP. de Holanda con $n=28$ publicaciones y 1157 citas, y Hegele, RA. de Canadá con $n=30$ publicaciones y 1003 citas asociadas a sus publicaciones. Discusión: La revisión indica producción importante en diagnóstico de HF en estos últimos diez años y progresos en el diagnóstico; sigue habiendo vacíos del conocimiento relacionados con la implementación de las políticas públicas, la falta de conocimiento sobre los riesgos de la HF en todos el personal interviniente y el desarrollo de las pruebas más efectivas y económicas.

Palabras Clave (DeCS/MeSH). Hyperlipoproteinemia Type II, diagnóstico, pruebas genéticas, mutación con pérdida de función, riesgo

\section{ABSTRACT.}

Familial Hypercholesterolemia ( $\mathrm{FH}$ ) is a genetic disorder that causes an increase in blood cholesterol and a significant increase in the development of atherosclerosis. Objective: To establish the state of research in the subject of $\mathrm{FH}$, to review the main documents developed in relation to the subject and to explore the possible gaps in knowledge 
that arise from this academic exercise with respect to diagnosis. Methods: A review was made with bibliometric indicators from articles of the Web of Science database, for the analysis the free programs VOSviewer and Bibliometrix application of R were used. Additionally, the most cited texts were reviewed. Results: A total of 1102 articles were found using the inclusion criteria. The most cited authors were Watts, GF. from Australia with $n=32$ publications and 1279 citations, followed by Kastelein, J.J. P. from Holland with $n=28$ publications and 1157 citations, and Hegele, RA. from Canada with $n=30$ publications and 1003 citations associated with their publications. Discussion: The review indicates important production in $\mathrm{FH}$ diagnosis in the last ten years and progress in diagnosis; there are still gaps in knowledge related to the implementation of public policies, lack of knowledge about the risks of $\mathrm{FH}$ in all the staff involved and the development of the most effective and economical tests.

Keywords (DeCS/MeSH). Hyperlipoproteinemia Type II, diagnosis, genetic testing, loss of function mutation, risk

\section{INTRODUCCIÓN.}

La Hipercolesterolemia Familiar (HF) es una condición autosómica dominante frecuente en el mundo (1). Las cifras indican que cerca de 34 millones de personas padecen este trastorno con una prevalencia calculada entre 1 de cada 250 y 1 de cada 313 individuos, rango que se explica por diferencias que favorecen su aparición a partir de la edad, y la ubicación geográfica $(2,3)$. Estas cifras señalan la importancia de este trastorno en el mundo e indican también una preocupación creciente por lograr un diagnóstico oportuno y un manejo temprano para impactar a la baja las cifras de riesgo en enfermedad cardiovascular temprana dado que se estima en algunos estudios que el diagnóstico no supera el $1 \%$, lo que indica que la mayoría de los pacientes permanecen sin diagnóstico (4).

El trastorno corresponde a varias formas de anormalidades genéticas que se heredan y que cursan con una concentración elevada del colesterol en suero. Normalmente los hallazgos clínicos tienen relación 
con niveles altos de colesterol de lipoproteínas de baja densidad (LDL) y básicamente puede tener dos presentaciones: La primera, que es la más común, es debida a una condición autosómica dominante, lo que quiere decir que la mitad de su descendencia también está afectada, y la segunda a una transmisión codominante con un $90 \%$ más de penetrancia (5). Esta situación genética induce en el individuo la aparición de aterosclerosis de inicio temprano y enfermedad cardiovascular que aumenta el riesgo de muerte en un $30 \%$ para las mujeres sobre los 60 años y en $50 \%$ para los hombres sobre los 50 años cuando no son tratados a tiempo (6).

Tres grupos de mutaciones genéticas frecuentes en HF han sido las más comúnmente reportadas, estas son, las propias del receptor de LDL, más de 1600 , que corresponden al $85 \%$ a $90 \%$ de los casos; las de la apolipoproteína B (ApoB) con cerca del $10 \%$ de los casos; y las de la subtisilina/Kexina tipo 9 de la proteína convertasa (PCSK9) con cerca del $5 \%$ de los casos ( 7 ); todas conducen a la alteración de los receptores de LDL y a una reducción de la captación de esta forma de colesterol con las consecuencias ya señaladas. Adicionalmente, si el defecto es homocigótico o heterocigótico determina la gravedad de la presentación y la edad de las manifestaciones para enfermedad cardiovascular. En caso de portadores de ambos genes alterados desde dos padres heterocigotos, los receptores son escasos y no hay como captar LDL por lo que se convierten en pacientes con hipercolesterolemia y enfermedad cardiovascular de inicio temprano que pueden morir antes de los 20 años si no reciben tratamiento (8).

En este sentido, el propósito del presente documento es el de establecer el estado de la investigación en el tema de HF y diagnóstico, revisar los principales documentos desarrollados con relación a la temática y explorar los posibles vacíos de conocimiento que surjan de este ejercicio académico con respecto al diagnóstico.

\section{METODOLOGÍA.}

Tipo de investigación: Se realizó una revisión con indicadores bibliométricos y se analizó adicionalmente los textos más citados al 
respecto para establecer intereses y posibles vacíos en el conocimiento con respecto al tema de HF.

\section{Selección de estudios y extracción de los datos}

Para los artículos se utilizó la información disponible en la base de datos de Web of Science (WoS) de Clarivate Analytics realizada el día 09 de diciembre de 2020. Para esto, se pusieron a prueba 4 ecuaciones de búsqueda con términos en inglés, de las cuales se estableció el uso de "Familial hypercholesterolemia" AND Diagnos* para recuperar la mayor cantidad de artículos en este tema. Se realizó la búsqueda por temas y además por títulos lo que arrojó dos archivos complementarios para el análisis.

Se incluyeron artículos en inglés, sin restricción por tipo de artículo, para la ventana de tiempo de 2009 a 2020, en todas las categorías de búsqueda en las que se clasifican los documentos WoS. La información bibliométrica utilizada partió desde los indicadores de producción de publicaciones, citas, autores más productivos y cooperación entre autores, co-ocurrencia desde los títulos y los resúmenes. Adicionalmente, se seleccionaron, por la cantidad de citas, un grupo de artículos considerados como los más relevantes en el tema de HF y diagnóstico, que fueron leídos a texto completo para realizar una revisión temática de contenidos.

\section{Análisis de los datos.}

Para el procesamiento de la información se utilizó los programas VOSviewer versión 1.6.14 (9) y la aplicación Bibliometrix de R en su versión 3.0.3 (10). Los dos permitieron representaciones de los datos cuantitativos de documentos, autores y términos, mediante la representación gráfica de los datos. 


\section{DISCUSIÓN Y RESULTADOS PARCIALES.}

\section{Producción anual de publicaciones.}

Los criterios de búsqueda permitieron establecer un conjunto de $n=1102$ artículos en el registro de temas y $n=155$ que contienen las palabras de la ecuación en el título para la ventana de tiempo establecida entre 2009 y 2020.

El promedio de artículos por año fue de $92 \pm 51$ artículos, con una mediana de 74. La curva indica que se produjo un crecimiento exponencial de publicaciones entre los años 2014 a 2018 en la temática. Cuando se observa la presencia de HF y diagnóstico presentes en el título, la mayor producción se aprecia entre los años 2013 y 2019 con un promedio de $n=13 \pm 7$ artículos. Ver figura 1 .

Figura 1. Número de publicaciones por año y valores acumulados para el periodo de 2009 a 2020. A la izquierda para HF y diagnóstico como tema y a la derecha para HF y diagnóstico presente en el título.
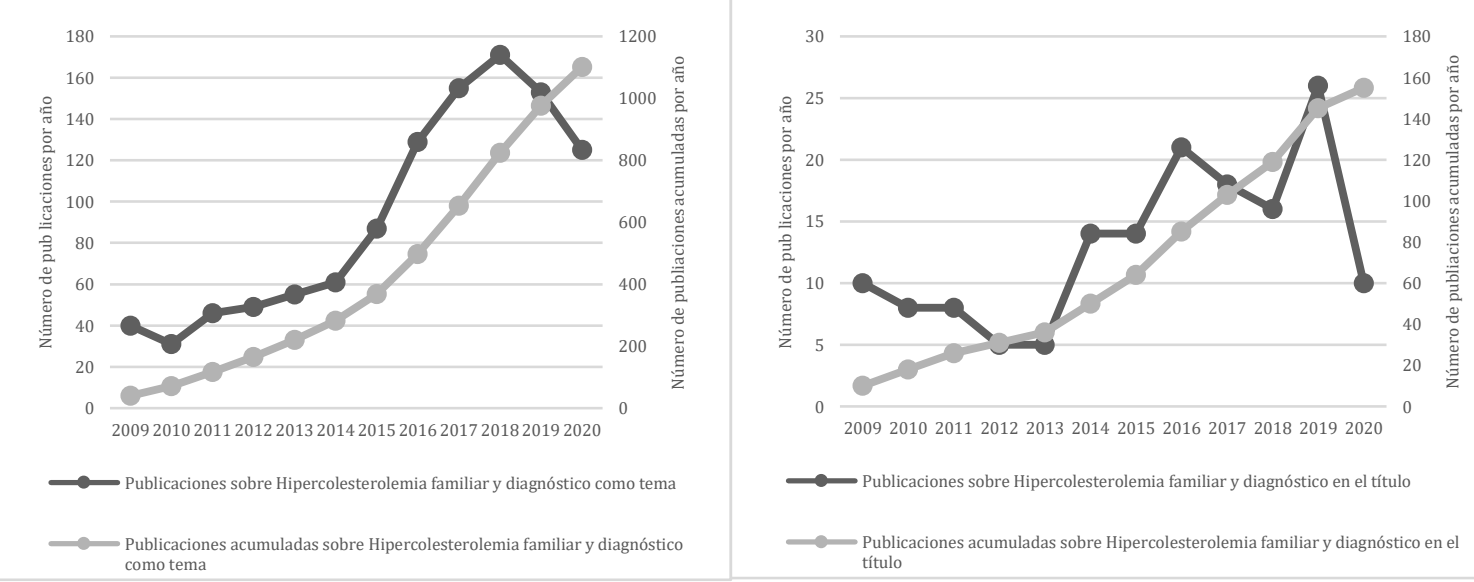

Autores más productivos, cooperación entre autores y temáticas. 
Un total de $n=4987$ autores fueron registrados cuando se ve el análisis por tema y $n=795$ cuando se revisa por el título. Para el caso por tema, los tres autores más productivos en este periodo de tiempo fueron, Watts, Gerald F. de Australia con $n=32$ publicaciones que tiene asociadas 1279 citas, seguido por Kastelein, John J. P. de Holanda con $\mathrm{n}=28$ publicaciones y $\mathrm{n}=1157$ citas, y Hegele, Robert A. de Canadá con $\mathrm{n}=30$ publicaciones y 1003 citas asociadas a sus publicaciones. Cuando se observa por título son los más productivos: Hopkins, Paul N. con $n=$ 5 artículos y 660 citas, Rader, Daniel J. con $n=4$ artículos asociado a $n=649$ citas y Ballantyne, Christie $M$. con $n=3$ artículos y $n=643$ citas; todos ellos de Estados Unidos. Ver tabla 1.

Tabla 1. Primeros 10 autores más productivos en el tema de Hipercolesterolemia familiar y diagnóstico.

\begin{tabular}{|c|c|c|c|c|c|}
\hline $\begin{array}{l}\text { Núme } \\
\text { ro }\end{array}$ & $\begin{array}{c}\text { Autores más } \\
\text { productivos en } \\
\text { el tema } \\
\text { hipercolesterole } \\
\text { mia familiar y } \\
\text { Diagnóstico por } \\
\text { tema }\end{array}$ & $\begin{array}{l}\text { País del } \\
\text { autor }\end{array}$ & $\begin{array}{c}\text { Número } \\
\text { de } \\
\text { publicacio } \\
\text { nes }\end{array}$ & $\begin{array}{c}\text { Cita } \\
\text { s }\end{array}$ & $\begin{array}{c}\text { Número } \\
\text { de } \\
\text { publicacio } \\
\text { nes como } \\
\text { primer } \\
\text { autor }\end{array}$ \\
\hline 1 & Watts, Gerald F. & Australia & 32 & $\begin{array}{c}127 \\
9\end{array}$ & 3 \\
\hline 2 & $\begin{array}{c}\text { Kastelein, John J. } \\
\text { P. }\end{array}$ & Holanda & 28 & $\begin{array}{c}115 \\
7\end{array}$ & 1 \\
\hline 3 & Hegele, Robert A. & Canadá & 30 & $\begin{array}{c}100 \\
3\end{array}$ & 5 \\
\hline 4 & Rader, Daniel J. & $\begin{array}{l}\text { Estados } \\
\text { Unidos }\end{array}$ & 11 & 943 & 1 \\
\hline 5 & Alonso, Rodrigo & Chile & 23 & 916 & 4 \\
\hline 6 & Santos, Raul D. & Brasil & 24 & 912 & 6 \\
\hline 7 & Hovingh, G. Kees & Holanda & 19 & 869 & 2 \\
\hline 8 & $\begin{array}{l}\text { Nordestgaard, } \\
\text { Borge G. }\end{array}$ & Dinamarca & 6 & 857 & 1 \\
\hline 9 & Kindt, Iris & $\begin{array}{l}\text { Estados } \\
\text { Unidos }\end{array}$ & 14 & 837 & 1 \\
\hline
\end{tabular}


El 20,9\% $(n=1043)$ de los autores no tienen citas asociadas a su producción. Mientras que el 5,4\% $(n=269)$ tienen más de 100 citas asociadas a sus documentos producidos.

Por otra parte, la cooperación entre autores permite establecer 10 clústeres de trabajo en la red para autores con mínimo 5 publicaciones. Ver figura 2.

Figura 2. Red de cooperación entre autores para el tema de HF y diagnóstico.

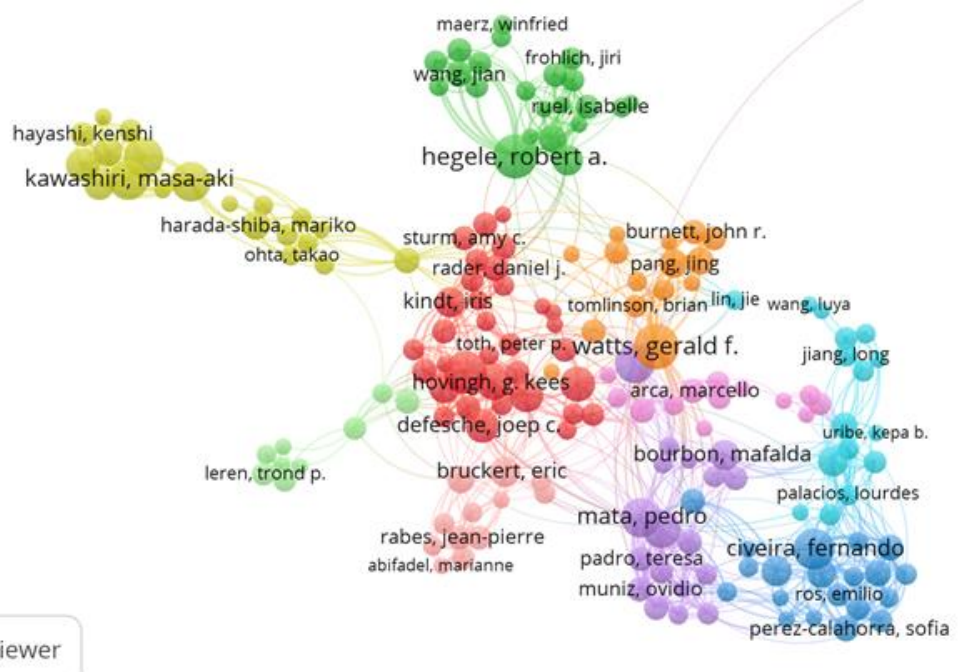

\section{Investigación por países}

Un total de 78 países se encuentra en los registros obtenidos para investigación en HF y diagnóstico. De estos, $\mathrm{n}=44$ países han participado en la construcción de más de 5 artículos. Estados Unidos con $\mathrm{n}=231$ artículos y $\mathrm{n}=6749$ citas es el país que encabeza la producción 
de sus investigadores, seguido por autores de España con $n=125$

publicaciones y $n=2208$ citas e Italia con $n=88$ artículos y $n=1552$ citas, Ver figura 3.

Figura 3. Distribución de publicaciones por país sobre el tema hipercolesterolemia familiar y diagnóstico. Gráfico con los 10 países de mayor producción.

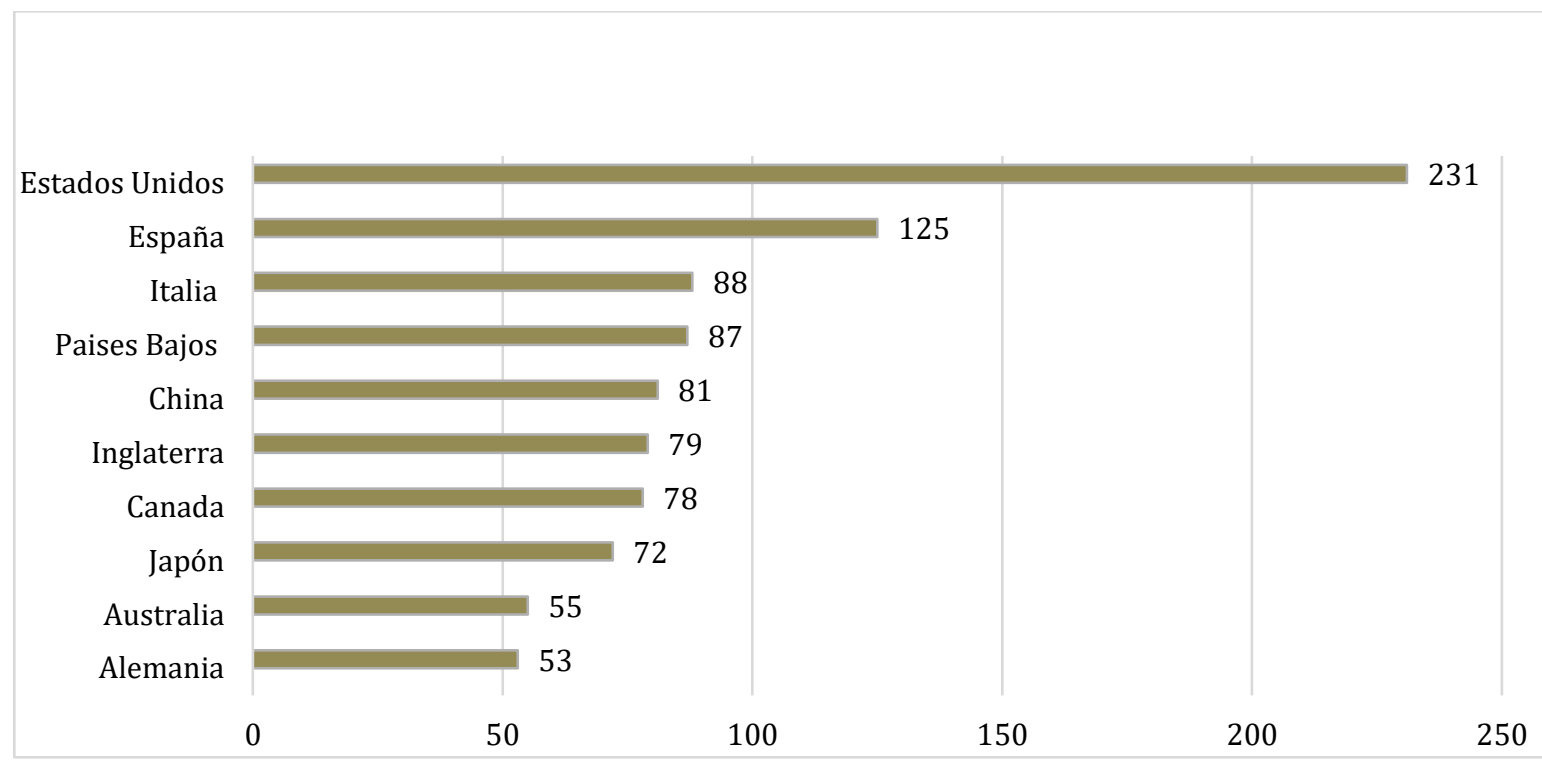

Cuando se observan los resultados por la presencia en el título de las palabras HF y diagnóstico se establecen como los primeros tres países por la cantidad de publicaciones a Estados Unidos con $n=151$ publicaciones, España con $n=97$ publicaciones y Reino Unido con $n=54$ publicaciones.

\section{Coocurrencia de palabras clave}

4 grupos de palabras se relacionan desde los artículos publicados para el tema de estudio, de los cuales $n=663$ cumplen con el criterio de selección de citarse en 10 o más publicaciones. En el primer grupo, de color rojo se establecen las palabras: estatinas, guías, Inhibidores, evento cardiovascular, cuidados, eficacia seguridad y medicamento; en 
el segundo grupo en verde, destacan las palabras: mutación, gene, variante, LDLR, ApoB, Familia, receptor, diagnóstico genético, diagnóstico clínico, causas genéticas, mecanismos, muestra, genotipo; en un tercer grupo de color azul las palabras que muestran frecuencias importantes son: Infarto de miocardio, hipertensión, edad, sexo, factor de riesgo cardiovascular, intervalo de confianza, Odds Ratio; y finalmente, en amarillo el grupo 3 de artículos que menciona el termino: Parientes de primer grado como el más frecuente. Ver figura 4.

Figura 4. Red de coocurrencia de palabras clave para el tema de HF y diagnóstico en títulos y resúmenes.

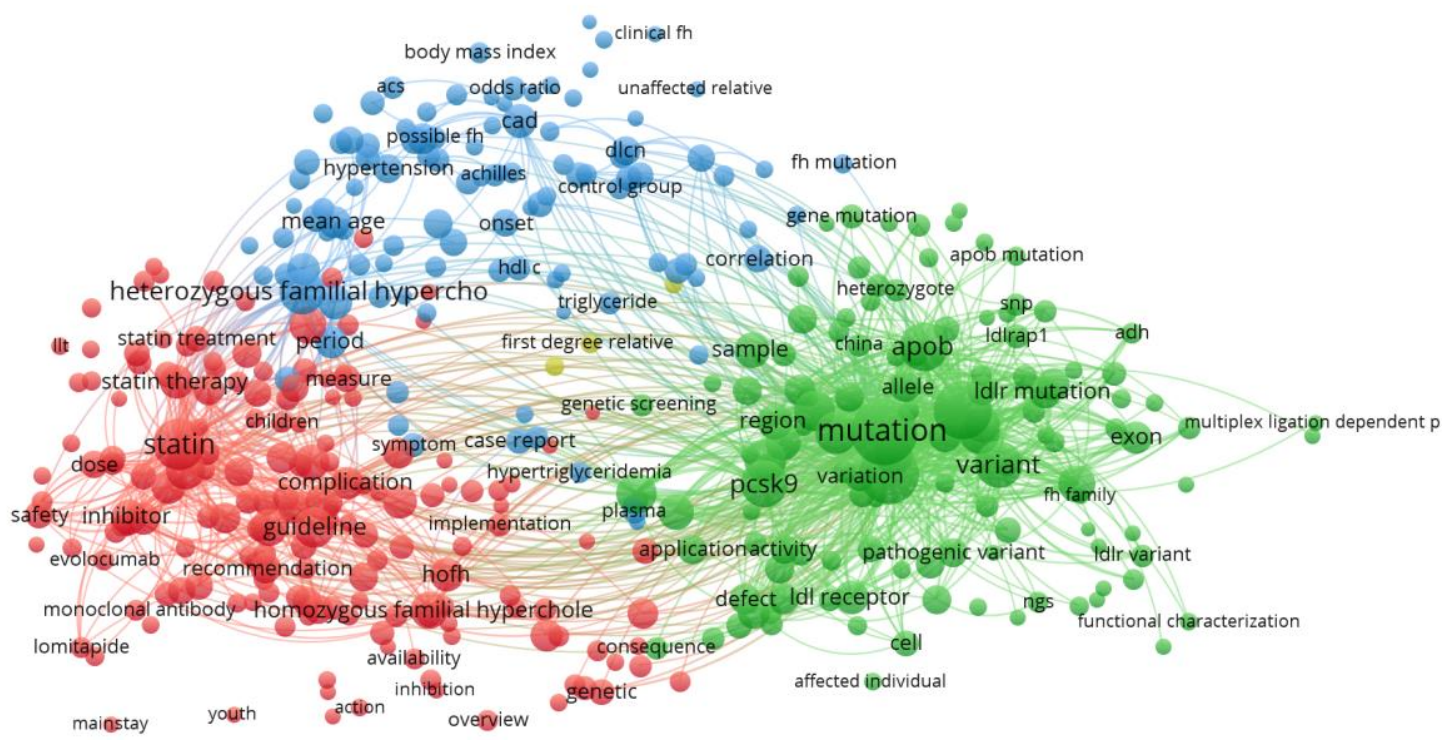

\& VOSviewer

En orden los 20 términos, en la producción por temática, que aparecen con mayor frecuencia son: mutación $(n=327)$, gene $(n=262)$, LDLR $(n=242)$, ApoB $(n=226)$, estatina $(n=170)$, variante $(n=143)$, PCSK9 $(n=139)$, HF heterozigótica $(n=124)$, Familia $(n=111)$, fenotipo $(n=108)$, paraproteína convertasa subtilisina $(n=99)$, Guías $(n=99)$, diagnóstico clínico $(n=93)$, Receptor de lipoproteína de baja densidad $(n=91)$, reducción $(n=89)$, medicamento $(n=84)$, cuidado $(n=80)$, gene $\operatorname{LDLR}(n=76)$, evento cardiovascular $(n=76)$ e HF homocigótica $(n=74)$. 
Para el caso de los artículos que contiene las palabras HF y diagnóstico en el título las 10 más frecuentes palabras son: Mutación $(n=24)$; enfermedad coronaria $(n=19)$; enfermedad $(n=19)$; guías $(n=18)$; gestión $(n=17)$; riesgo $(n=16)$; Enfermedad cardiovascular $(n=15)$; prevalencia $(n=15)$; hipercolesterolemia autosómico dominante $(n=14)$ y población $(n=13)$. Ver figura 5 .

Figura 5. Mapa de ocurrencia de palabras en artículos que contienen en el título HF y diagnóstico.

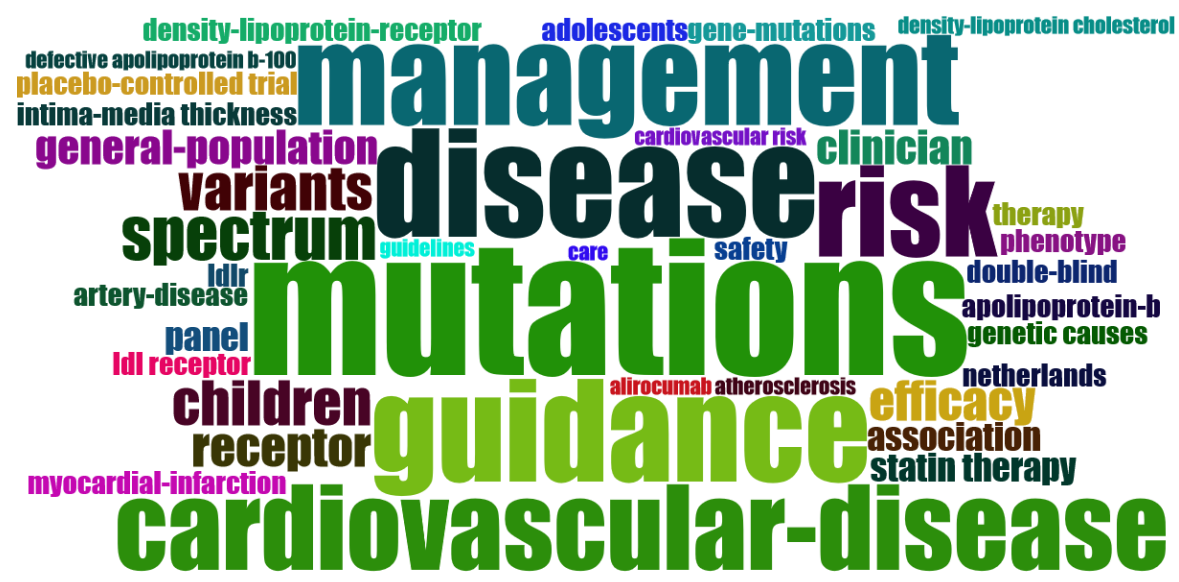

Figura 6. Red de autores citados con relación al tiempo para HF y diagnóstico. 


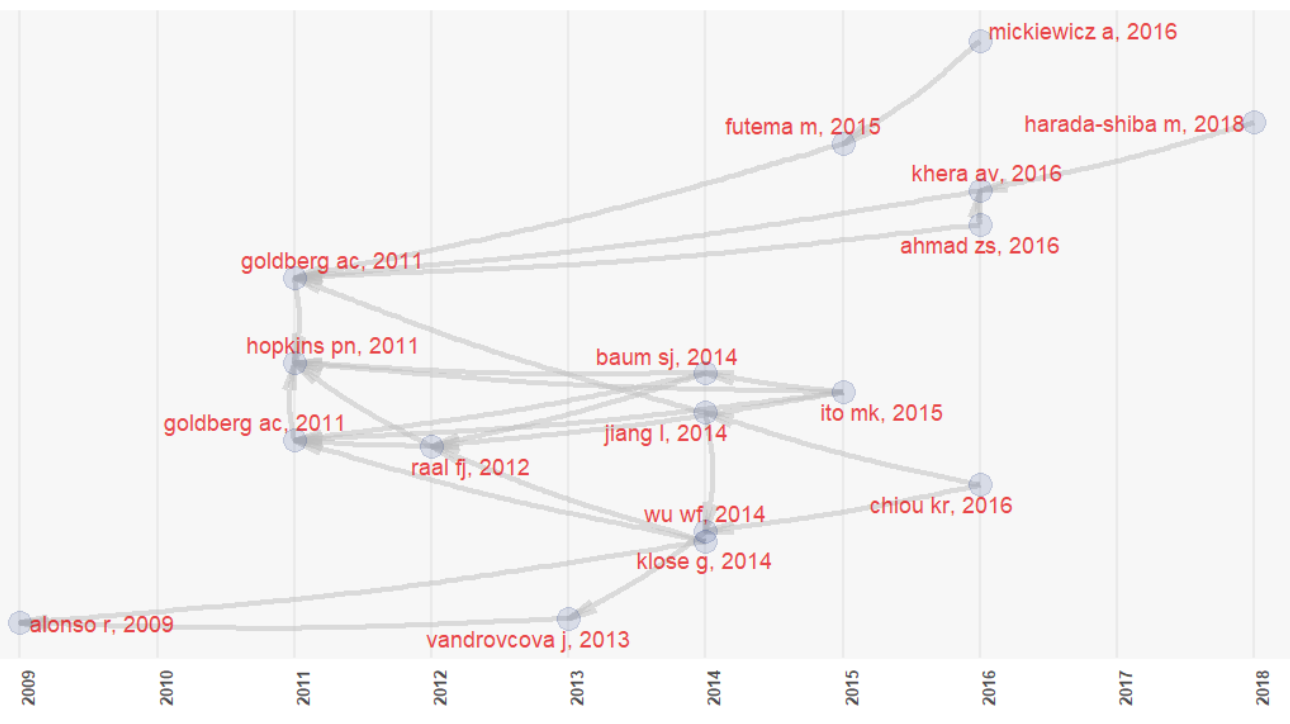

Tabla 2. Artículos con mayor cantidad de citas por título para Hipercolesterolemia Familiar y Diagnóstico.

\begin{tabular}{|c|c|c|c|c|c|}
\hline No. & $\begin{array}{l}\text { Título del } \\
\text { artículo }\end{array}$ & Autor(es) & $\begin{array}{l}\text { Journal y F. } \\
\text { de impacto }\end{array}$ & $\begin{array}{l}\text { Año } \\
\text { de } \\
\text { publi } \\
\text { cació } \\
\text { n }\end{array}$ & Citas \\
\hline 1 & $\begin{array}{l}\text { Familial } \\
\text { Hypercholesterole } \\
\text { mia: Screening, } \\
\text { diagnosis and } \\
\text { management of } \\
\text { pediatric and } \\
\text { adult patients. } \\
\text { Clinical guidance } \\
\text { from the National } \\
\text { Lipid Association } \\
\text { Expert Panel on } \\
\text { Familial } \\
\text { Hypercholesterole } \\
\text { mia }\end{array}$ & $\begin{array}{c}\text { Anne C } \\
\text { Goldberg, } \\
\text { Paul N } \\
\text { Hopkins, } \\
\text { Peter P Toth, } \\
\text { William C. } \\
\text { Cromwell, } \\
\text { Joyce L. } \\
\text { Ross, Paul E. } \\
\text { Ziajka }\end{array}$ & $\begin{array}{c}\text { Journal of } \\
\text { Clinical } \\
\text { Lipidology } \\
(3.860)\end{array}$ & 2011 & 439 \\
\hline 2 & $\begin{array}{l}\text { Diagnostic Yield } \\
\text { and Clinical Utility } \\
\text { of Sequencing } \\
\text { Familial } \\
\text { Hypercholesterole } \\
\text { mia Genes in }\end{array}$ & $\begin{array}{l}\text { Amit } \\
\text { V.Khera, } \\
\text { Hong- } \\
\text { HeeWon, } \\
\text { Gina } \\
\text { M.Peloso, }\end{array}$ & $\begin{array}{c}\text { Journal of } \\
\text { the American } \\
\text { College of } \\
\text { Cardiology } \\
(20.859)\end{array}$ & 2016 & 327 \\
\hline
\end{tabular}




\begin{tabular}{|c|c|c|c|c|c|}
\hline & $\begin{array}{l}\text { Patients with } \\
\text { Severe } \\
\text { Hypercholesterole } \\
\text { mia }\end{array}$ & $\begin{array}{c}\text { Kim } \\
\text { S.Lawson. }\end{array}$ & & & \\
\hline 3 & $\begin{array}{l}\text { Familial } \\
\text { Hypercholesterole } \\
\text { mias: Prevalence, } \\
\text { genetics, } \\
\text { diagnosis and } \\
\text { screening } \\
\text { recommendations } \\
\text { from the National } \\
\text { Lipid Association } \\
\text { Expert Panel on } \\
\text { Familial } \\
\text { Hypercholesterole } \\
\text { mia }\end{array}$ & $\begin{array}{l}\text { Paul N. } \\
\text { Hopkins, } \\
\text { Peter P. } \\
\text { Toth, } \\
\text { Christie M. } \\
\text { Ballantyne y } \\
\text { Daniel J. } \\
\text { Rader, }\end{array}$ & $\begin{array}{l}\text { Journal of } \\
\text { Clinical } \\
\text { Lipidology } \\
(3.860)\end{array}$ & 2011 & 207 \\
\hline 4 & $\begin{array}{l}\text { Homozygous } \\
\text { familial } \\
\text { hypercholesterole } \\
\text { mia: Current } \\
\text { perspectives on } \\
\text { diagnosis and } \\
\text { treatment }\end{array}$ & $\begin{array}{l}\text { Frederick J. } \\
\text { Raal y Raul } \\
\text { D. Santos }\end{array}$ & $\begin{array}{l}\text { Atherosclero } \\
\text { sis }(3.919)\end{array}$ & 2012 & 191 \\
\hline 5 & $\begin{array}{l}\text { Refinement of } \\
\text { Variant Selection } \\
\text { for the LDL } \\
\text { Cholesterol } \\
\text { Genetic Risk } \\
\text { Score in the } \\
\text { Diagnosis of the } \\
\text { Polygenic Form of } \\
\text { Clinical Familial } \\
\text { Hypercholesterole } \\
\text { mia and } \\
\text { Replication in } \\
\text { Samples from } 6 \\
\text { Countries }\end{array}$ & $\begin{array}{c}\text { Marta } \\
\text { Futema, } \\
\text { Sonia Shah, } \\
\text { Jackie A. } \\
\text { Cooper, } \\
\text { KaWah Li, Et } \\
\text { al. }\end{array}$ & $\begin{array}{c}\text { Clinical } \\
\text { Chemistry } \\
(7.292)\end{array}$ & 2015 & 113 \\
\hline 6 & $\begin{array}{l}\text { Two Years after } \\
\text { Molecular } \\
\text { Diagnosis of } \\
\text { Familial } \\
\text { Hypercholesterole } \\
\text { mia: Majority on }\end{array}$ & $\begin{array}{l}\text { Roeland } \\
\text { Huijgen, Iris } \\
\text { Kindt, Sjoerd } \\
\text { B J } \\
\text { Verhoeven, }\end{array}$ & $\begin{array}{l}\text { Plos One } \\
(2.740)\end{array}$ & 2010 & 95 \\
\hline
\end{tabular}




\begin{tabular}{|c|c|c|c|c|c|}
\hline & $\begin{array}{l}\text { Cholesterol- } \\
\text { Lowering } \\
\text { Treatment but a } \\
\text { Minority Reaches } \\
\text { Treatment Goal }\end{array}$ & $\begin{array}{c}\text { Eric J G } \\
\text { Sijbrands, } \\
\text { Maud N } \\
\text { Vissers, John } \\
\text { J P Kastelein, } \\
\text { Barbara A } \\
\text { Hutten. }\end{array}$ & & & \\
\hline 7 & $\begin{array}{l}\text { Use of Targeted } \\
\text { Exome } \\
\text { Sequencing in } \\
\text { Genetic Diagnosis } \\
\text { of Chinese } \\
\text { Familial } \\
\text { Hypercholesterole } \\
\text { mia }\end{array}$ & $\begin{array}{c}\text { Wen-Feng } \\
\text { Wu, Li-Yuan } \\
\text { Sun, Xiao- } \\
\text { Dong Pan, } \\
\text { Shi-Wei Yang } \\
\text { y Lv-Ya } \\
\text { Wang }\end{array}$ & $\begin{array}{c}\text { Plos One } \\
(2.740)\end{array}$ & 2014 & 86 \\
\hline 8 & $\begin{array}{l}\text { Perceived Risk } \\
\text { and } \\
\text { Representations } \\
\text { of Cardiovascular } \\
\text { Disease and } \\
\text { Preventive } \\
\text { Behaviour in } \\
\text { People Diagnosed } \\
\text { with Familial } \\
\text { Hypercholesterole } \\
\text { mia: A Cross- } \\
\text { sectional } \\
\text { Questionnaire } \\
\text { Study }\end{array}$ & $\begin{array}{c}\text { Liesbeth } \\
\text { Claassen, } \\
\text { Lidewij } \\
\text { Henneman, } \\
\text { Iris Kindt, } \\
\text { Theresa M. } \\
\text { Marteau, } \\
\text { Danielle R.M. } \\
\text { Timmermans }\end{array}$ & $\begin{array}{c}\text { Journal of } \\
\text { Health } \\
\text { Psychology } \\
(2.503)\end{array}$ & 2010 & 56 \\
\hline 9 & $\begin{array}{l}\text { The use of next- } \\
\text { generation } \\
\text { sequencing in } \\
\text { clinical diagnosis } \\
\text { of familial } \\
\text { hypercholesterole } \\
\text { mia }\end{array}$ & $\begin{array}{c}\text { Jana } \\
\text { Vandrovcova } \\
\text {, Ellen R.A. } \\
\text { Thomas, } \\
\text { Santosh S } \\
\text { Atanur, } \\
\text { Penny J. } \\
\text { Norsworthy. }\end{array}$ & $\begin{array}{c}\text { Genetics in } \\
\text { Medicine } \\
(8,904)\end{array}$ & 2013 & 49 \\
\hline
\end{tabular}

\section{CONCLUSIONES.}

Esta revisión señala que se ha hecho un esfuerzo en la investigación sobre HF y diagnóstico en estos últimos diez años, que ha logrado 
progresos en las mejoras de diagnóstico y que se ha avanzado en el llamado de atención a los entes gubernamentales y de salud pública con respecto al subdiagnóstico y las necesidades de reducción de la carga de enfermedad, sin embargo, siguen habiendo vacíos del conocimiento, que están relacionados con la implementación de las políticas públicas, la falta de conocimiento sobre los riesgos de la HF en todos el personal interviniente, el desarrollo de las más efectivas y económicas pruebas genéticas posibles, y la puesta en marcha de políticas que permitan derribar las barreras de accesos al diagnóstico y tratamiento de los grupos poblacionales afectados, con especial énfasis en las edades escolares.

\section{BIBLIOGRAFÍA.}

1. Cuchel M, Bruckert E, Ginsberg HN, Raal FJ, Santos RD, Hegele RA, et al. Homozygous familial hypercholesterolaemia: new insights and guidance for clinicians to improve detection and clinical management. A position paper from the Consensus Panel on Familial Hypercholesterolaemia of the European Atherosclerosis Society. Eur Heart J. 2014 Aug;35(32):2146-57.

2. Nordestgaard BG, Chapman MJ, Humphries SE, Ginsberg HN, Masana L, Descamps OS, et al. Familial hypercholesterolaemia is underdiagnosed and undertreated in the general population: guidance for clinicians to prevent coronary heart disease: consensus statement of the European Atherosclerosis Society. Eur Heart J. 2013 Dec;34(45):3478-90a.

3. Hu P, Dharmayat KI, Stevens CAT, Sharabiani MTA, Jones RS, Watts GF, et al. Prevalence of Familial Hypercholesterolemia Among the General Population and Patients With Atherosclerotic Cardiovascular Disease: A Systematic Review and Meta-Analysis. Circulation. 2020 May; 141(22):1742-59.

4. Akioyamen LE, Genest J, Shan SD, Reel RL, Albaum JM, Chu A, et al. Estimating the prevalence of heterozygous familial hypercholesterolaemia: a systematic review and meta-analysis. BMJ Open. 2017 Sep;7(9):e016461. 
5. Vaezi Z, Amini A. Familial Hypercholesterolemia. In Treasure Island (FL); 2020.

6. Kim YR, Han KH. Familial hypercholesterolemia and the atherosclerotic disease [Internet]. Vol. 43, Korean Circulation Journal. The Korean Society of Cardiology; 2013. p. 363-7. Available from: /pmc/articles/PMC3717417/?report=abstract

7. Susan-Resiga D, Girard E, Scott Kiss R, Essalmani R, Hamelin J, Asselin $\mathrm{M}-\mathrm{C}$, et al. The Proprotein Convertase Subtilisin/Kexin Type 9-resistant R410S Low Density Lipoprotein Receptor Mutation A NOVEL MECHANISM CAUSING FAMILIAL HYPERCHOLESTEROLEMIA S. 2016; Available from: http://www.jbc.org/

8. Mata $P$, Alonso R, Pérez-Jiménez F. Screening for Familial Hypercholesterolemia: a Model for Preventive Medicine. Rev Española Cardiol (English Ed [Internet]. 2014 Sep 1;67(9):685-8. Available from: http://www.revespcardiol.org/en-screening-forfamilial-hypercholesterolemia-model-articulo-S1885585714001212

9. Jeong D, Koo Y. Analysis of Trend and Convergence for Science and Technology using the VOSviewer. Int J Contents. 2016;12(3):54-8.

10. Aria M, Cuccurullo C. bibliometrix: An R-tool for comprehensive science mapping analysis. J Informetr. 2017 Nov 1;11(4):959-75.

11. Ray KK, Watts GF. Improving the global care of familial hypercholesterolaemia: Starting the ball rolling. Atherosclerosis [Internet]. 2019;277:230-3. Available from: https://doi.org/10.1016/j.atherosclerosis.2018.09.002

12. Jackson $\mathrm{CL}$, Keeton JZ, Eason SJ, Ahmad ZA, Ayers CR, Gore MO, et al. Identifying Familial Hypercholesterolemia Using a Blood Donor Screening Program with More Than 1 Million Volunteer Donors. JAMA Cardiol [Internet]. 2019 Jul 1;4(7):685-9. Available from: https://jamanetwork.com/

13. Knowles JW, O'Brien EC, Greendale K, Wilemon K, Genest J, Sperling LS, et al. Reducing the burden of disease and death from familial hypercholesterolemia: A call to action [Internet]. Vol. 168, American Heart Journal. Mosby Inc.; 2014 p. 807-11. Available from: /pmc/articles/PMC4683103/?report=abstract

14. Carlson B. Familial hypercholesterolemia captures gene test 
controversies. Biotechnol Healthc [Internet]. 2010;7(1):8-9. Available from: http://www.ncbi.nlm.nih.gov/pubmed/22478804 